\title{
Experiential learning placements in primary care: Responding to the COVID-19 pandemic
}

\author{
Alyson Brown, Linsey Hayes, Lloyd Kennett \\ Kirklands Hospital, NHS Lanarkshire, Lanarkshire, United Kingdom
}

\author{
Keywords \\ Experiential learning \\ Pharmacy education \\ Pharmacy student \\ Placement \\ Primary care \\ Undergraduate \\ Correspondence
Alyson Brown
Kirklands Hospital
NHS Lanarkshire
Lanarkshire, ML2 ODP
United Kingdom
alyson.brown2@lanarkshire.scot.nhs.uk
}

\section{Introduction}

Since 2018, Additional Cost of Teaching (ACT) Pharmacy funding has supported the development and delivery of experiential learning (EL) placements for undergraduate (UG) student pharmacists across Scotland (NES 2021), creating opportunities for EL placements to take place in a variety of practice settings, more closely aligned with the current pharmacy workforce. EL placements provide student pharmacists with opportunities to undertake tasks, such as medicines reconciliation, producing care plans, patient education, history taking, clinical skills, and consultation skills under supervision and supported by EL facilitators. The recently approved General Pharmaceutical Society (GPhC) Standards for the Initial Education and Training of Pharmacists (GPhC 2021) demonstrates a further move towards extended EL placements to support student pharmacists to be prescriber-ready by the end of the UG education, supported by an ambitious plan to increase EL placements to 11 weeks across the UG curriculum in Scotland. Primary care EL placements in Scotland are situated in the final two years of the UG course alongside EL placements in three other areas of practice (community, hospital, and specialist) and have associated Learning Outcomes defined by each university, such as understanding the role of the pharmacist in primary care, participating in counselling, history taking, and pharmaceutical care planning for patients.

In 2020, the Sars-2-CoV pandemic led to the need to reduce footfall in clinical settings, physical distancing measures, a blended approach to working location and a change to workflow in primary care settings in NHS Lanarkshire.

This short report focuses on how the primary care prescribing support team in NHS Lanarkshire adapted student pharmacist EL placements to ensure they continued to meet Learning Outcomes as defined by the universities via virtual delivery hosted on Microsoft (MS) Teams supported by the development of an EL Toolkit with a range of different activities included. 


\section{Description of programme \\ Development of Primary Care Toolkit}

The team understood the need for the EL placement to balance developing an appreciation of the role of a pharmacist working within the primary care setting with supported opportunities to learn, develop, and practice skills. Using the EL toolkit, the team created a schedule involving a series of underpinning seminars, simulated activities and debrief sessions (examples provided in Table I).

\section{Table I: Sample of NHS Lanarkshire primary care} toolkit activities

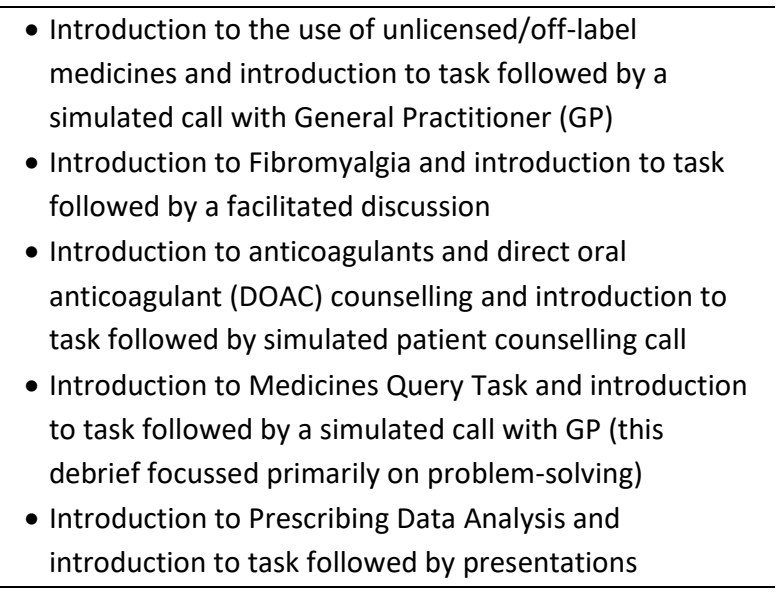

The virtual EL placements are delivered based on the following principles.

\section{Principles of simulation to create real-world experiences}

Plans to deliver an EL placement programme remotely raised questions around confidentiality, appropriate access to patient consultations and data, limited access to multidisciplinary team members, and, therefore, limited ability to carry out specific workplace tasks. To overcome this, a team of primary care and education and training pharmacists developed a series of simulated activities to create real-world experiences, giving student pharmacists opportunities to explore how they would respond within specific situations. Each simulated task was hosted by a facilitator, introducing a case study and suggestions for what the student pharmacist should consider during their preparation time for simulated consultations with the patient actor or simulated discussions with another health professional. Members of the pharmacy team played the simulated roles and provided feedback for the debrief that followed each simulated session

\section{Application of knowledge and skills to role-based activities}

The General Medical Services (GMS) contract in Scotland (Scottish Government, 2017) defines the role of the pharmacist in primary care through the delivery of a pharmacotherapy service with three levels of responsibility: core (level one), additional advanced (level two), and advanced specialist (level three). The tasks and activities developed for the toolkit focused on core level one pharmacotherapy activities and tasks associated with the broader prescribing support role, as these are the core activities of pharmacists in primary care and most closely aligned with early career pharmacist activity in this area of practice. Specialist seminars linked with level two and three activities were delivered during each of the EL placement days related to services delivered by the prescribing support team in NHS Lanarkshire.

\section{Consolidation of knowledge and extension of learning into the practice environment}

The last activity focused on the consolidation of knowledge and skills in the context of prescribing data analysis, allowing student pharmacists to carry out a task that would be routinely undertaken in practice. An initial presentation and brief were delivered, signposting student pharmacists to resources to support preparation for a presentation they would later deliver to a group of their peers. These were followed by a facilitated discussion exploring problem-solving and decision-making skills along with prioritising and presenting findings.

\section{Use of MS Teams}

An MS Teams 'team' was set up with all the facilitators and student pharmacists as members. Student pharmacists were informed of the MS Team at a preplacement induction seminar and given information on how the placement would run, and a schedule was then published on the MS Teams area in advance of the placement. All the seminars took place in the 'General' room while simulated calls were scheduled in specific channels, and a separate facilitator channel was included to provide support to facilitators as required throughout the placement.

\section{Initial feedback and future plans}

Across the programme, 95 student pharmacists attended a virtual primary care EL placement with NHS Lanarkshire. Initial feedback from both student pharmacists and facilitators was overwhelmingly 
positive, with good engagement with scheduled activities. Over 30 members of the primary care team were involved in the development and delivery of the EL toolkit across the academic session. The schedule was designed to ensure a balance of direct engagement along with time away from the screen, and this principle was continued for all virtual EL programmes delivered by NHS Lanarkshire in the 2020-21 academic session. It also enabled facilitators to be both involved in the delivery of the virtual EL programme and continue to meet operational needs during the pandemic. Facilitators (who had not been involved directly in the development of the toolkit) requested further support with the use of MS teams and the information provided within the toolkit, and the Education and Training team provided additional support to address this feedback. Further virtual EL placements for hospitals were delivered during January-May 2021 based on the principles described in this report to respond to local COVID-19 pressures. The toolkit has been further developed by a newly formed network of 'EL Champions' supported by the Education and Training team to focus on a series of activities, which will support placements in primary care through offering facilitators suggested tasks for use in the practice setting, with specific information provided on expected supervision for the task and key learning points.

\section{References}

General Pharmaceutical Council, 2021. Standards for the initial education and training of pharmacists [online]. London: GPhC. Available from: https://www.pharmacyregulation.org/sites/default/files/do cument/standards-for-the-initial-education-and-training-ofpharmacists-january-2021_0.pdf [Accessed 27 April 2021]

NES, 2021. Experiential Learning for Student Pharmacists in Scotland. [online]. Edinburgh: NES. Available from: https://www.nes.scot.nhs.uk/our-work/experientiallearning-for-student-pharmacists-in-scotland/ [Accessed 27 April 2021]

Scottish Government, 2017. GMS Contract: 2018 [online]. Edinburgh: Scottish Government. Available from: https://www.gov.scot/publications/gms-contract-scotland/ [Accessed 17 April 2021] 